\title{
AGE-RELATED DRIVING PERFORMANCE: EFFECT OF FOG UNDER DUAL-TASK CONDITIONS
}

\author{
Rui Ni, Julie Kang, and George J. Andersen \\ Department of Psychology \\ University of California Riverside \\ Riverside, California, USA \\ E-mail: ruini@ucr.edu
}

\begin{abstract}
Summary: The present study investigated the driving performance of older and younger drivers using a dual-task paradigm. Drivers were requred to do a car-following task while detecting a signal light change in a light array above the roadway in the driving simulator under different fog conditions. Decreased accuracies and longer response times were recorded for older drivers, compared to younger drivers, expecially under dense fog conditions. In addition, older drivers had decreased car following performance when simultaneously performing the light-detection task. These results suggets that under poor weather conditions (e.g. fog), with reduced visibility, older drivers may have an increased accident risk because of a decreased ability to perform multiple tasks.
\end{abstract}

\section{INTRODUCTION}

Previous research has documented age-related decrements in visual performance. These studies have included driving-related tasks such as steering control and collision detection (Andersen \& Sauer, 2004; Ni, Andersen, and Rizzo, 2005; Andersen \& Enriquez, 2006). Other studies have shown performance decrements when performing multiple tasks (Kramer \& Larish, 1996). The objectives of the present study were twofold. First, to examine the age-related effects of performing multiple driving tasks, and second, to determine whether simulated inclement weather (i.e., fog) will differentially affect the performance of older drivers because of reduced contrast of the driving scene.

Driving simulation displays depicted a roadway scene of city blocks with traffic in two adjacent lanes and a vehicle located directly in front of the driver's vehicle. Drivers were asked to perform a car-following task and to detect a light change in an array of lights located above the roadway. For the car-following task drivers were shown an initial period of constant speed and fixed distance behind the lead vehicle. After the initial period, the lead vehicle speed changed according to a sum of 3 sine wave functions, and drivers were asked to maintain the initial driving distance. When the driver's vehicle arrived at a pre-specified distance from the light array, one of the lights changed and drivers were asked to indicate whether the light change occurred on the left or right side of the array. To measure the effect of foggy weather, we systematically varied the fog condition from clear (i.e., no fog) to dense fog (shown in Figure 1). 


\section{Experiment}

Drivers. Eight older drivers (mean age $=75$ ) and 8 younger drivers (mean age $=23$ ) paticipated in a dual-task performance study using a driving simulator. All drivers had a minimum of 2 years of driving experience, had normal or corrected to normal vision, and were naïve to the purpose of the experiment.

Apparatus. The displays were presented on a Dell PC computer system. The display has a visual angle of $47 \mathrm{deg}$ by $26 \mathrm{deg}$, with the refresh rate at $60 \mathrm{~Hz}$ and the resolution at 1024 by 768 . A Logitech Wingman FomulaGP control system, including acceleration and brake pedals, was used for closed-loop control of the simulator. Drivers viewed the displays binocularly at a distance of approximately $60 \mathrm{~cm}$ from the screen.

Stimuli. The driving simulation depicted a roadway scene of city blocks with traffic in two adjacent lanes and a vehicle located directly in front of the driver's vehicle. An array of signal lights either in red or green was located above the roadway. The array consisted of 21 lights with 10 lights on the right, 10 on the left and one aligned with the center of the driver's position. The light change occurred at either the 3rd, 6th, or 9th light location from the center light ( 0 position) and occurred on either the right or left side.

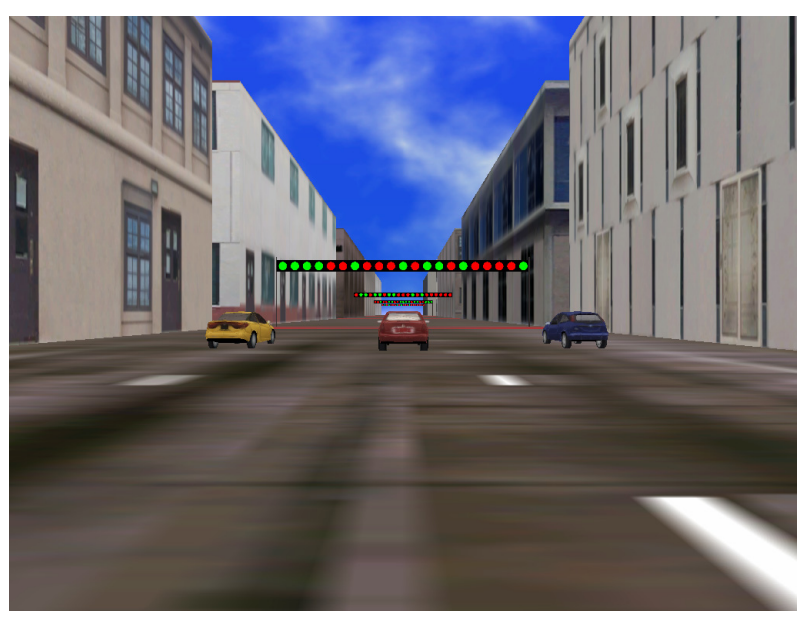

a

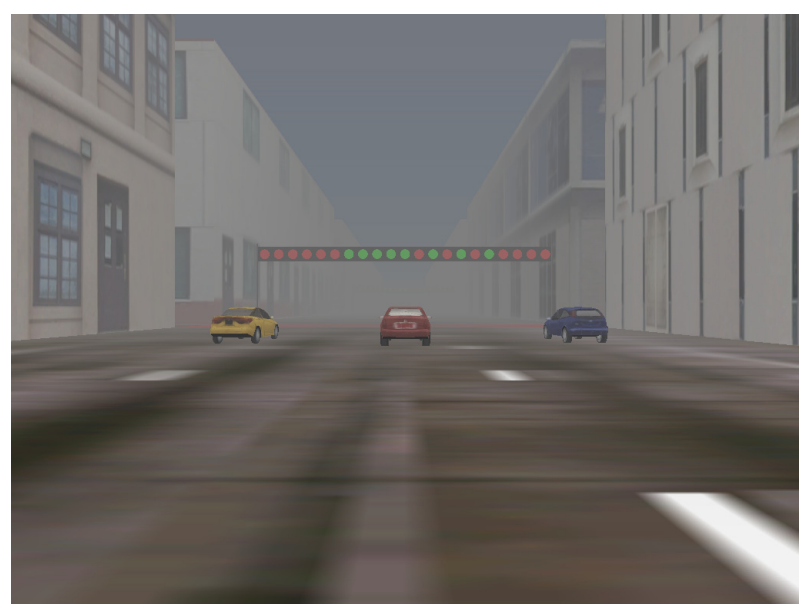

$\mathrm{b}$

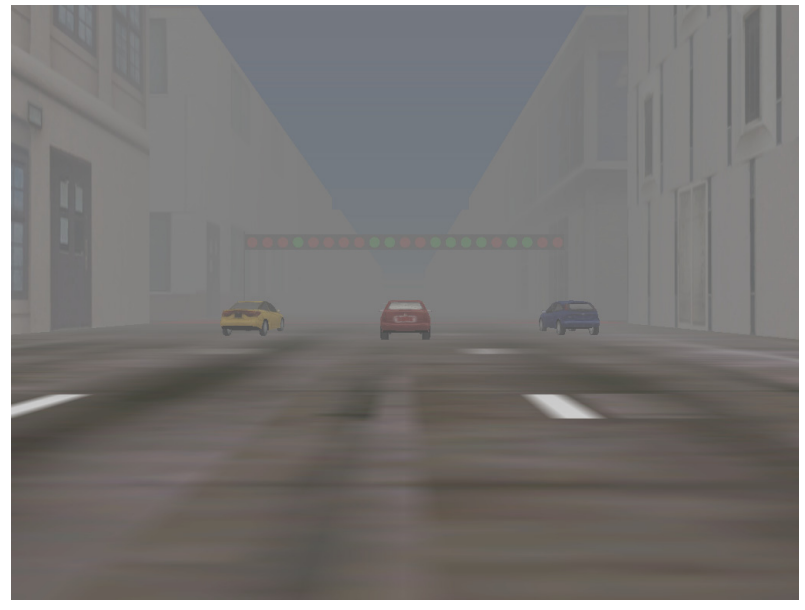

c

Figure 1. Three fog conditions in the experiment. a, no fog; $b$, medium fog; and c, dense fog. 
Design. The independent variables were fog density (no fog, medium fog, and dense fog), the horizontal position of the light when a change occurred (3rd, 6th or 9th position), and the location of the light change (left or right). These variables were run as within-subjects variables. Age group (younger vs. older) was run as a between-subjects variable. The dependent variables were accuracy and response time (for the light-change detection task), and speed difference between the driver's vehicle and the lead vehicle (for the car-following task).

Procedure. Two tasks were examined: car following and detecting a light change. For the car-following task, drivers were required to keep a constant distance behind the lead vehicle, which varied its speed after an initial period of travelling. For the light-detection task, drivers were asked to respond to a light change in an array of lights located above the roadway. Drivers performed both single and dual-task conditions. No fog was simulated in the single-task baseline conditions and the light-change task involved a change at the 3rd position. In the other sessions, 3 fog conditions were employed: no fog, medium fog, and dense fog.

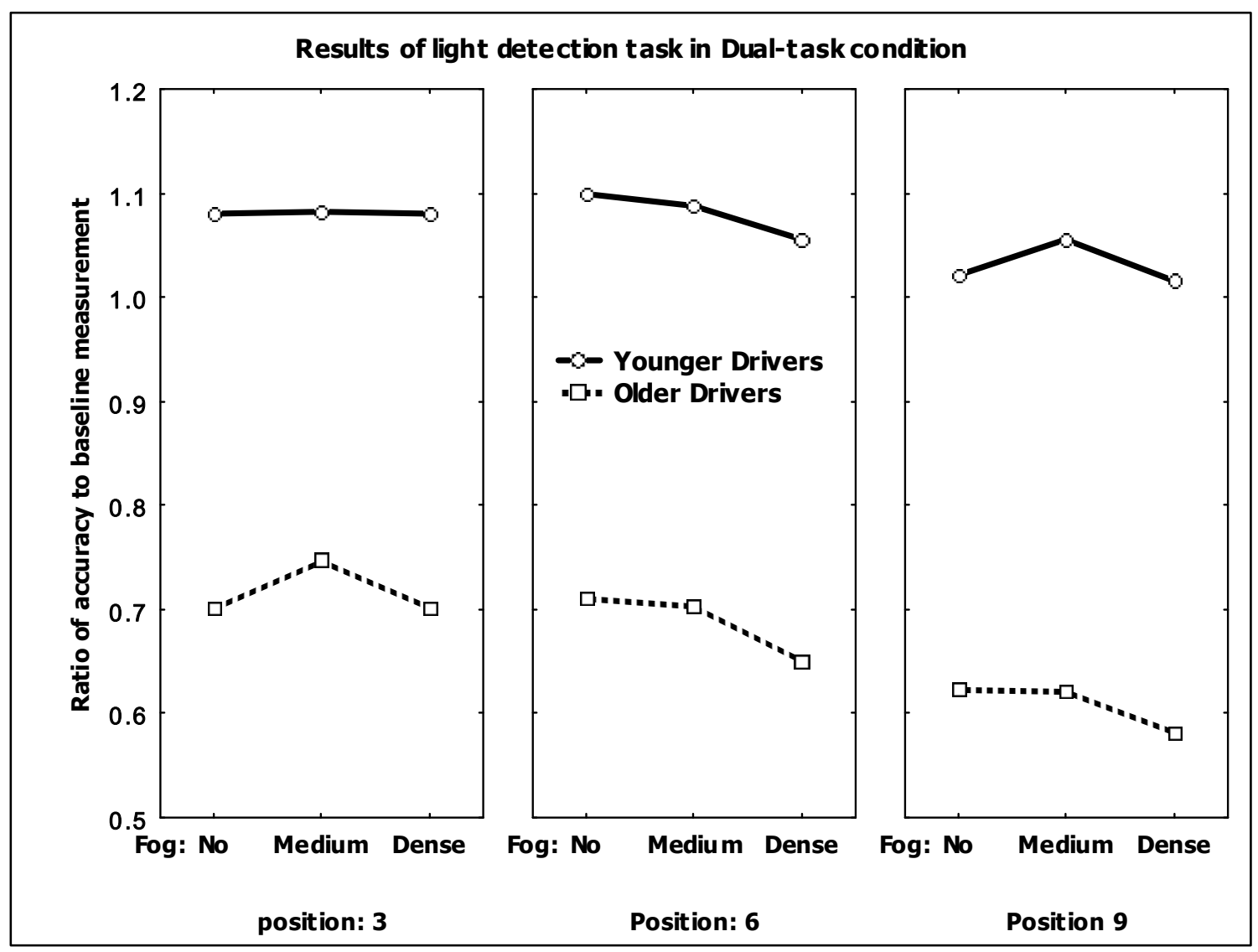

Figure 2. The performance on light-detection task in dual-task condition for two age groups. The dependent variable is the ratio of judgment accuracy in dual-task condition to that in baseline condition. 


\section{RESULTS}

For the light-detection task a 2 (task condition, i.e., single, and dual-task) by 3 (fog condition) by 3 (light change position) analysis of variance (ANOVA) was used to analyze mean accuracy and response time for each driver in the two age groups. With regard to accuracy (shown in Figure 2), the main effect of task condition (single vs. dual) was significant $(F(1,14)=15.661, p<0.01)$. Significant differences were also found between younger and older drivers $(F(1,14)=9.4023$, $\mathrm{p}<0.01)$. The results showed that accuracy was greater in the single-task than in dual-task condition and was greater for younger drivers as compared to older drivers. Significant interactions were also found between task condition and age group $(F(1,14)=6.2360, p<0.05)$, between task condition and light-change position $(\mathrm{F}(2,28)=4.9903, \mathrm{p}<0.05)$, between task condition, fog density and light-change position $(\mathrm{F}(4,56)=2.8796, \mathrm{p}<0.05)$, and between task condition, fog density, light-change position, and age group $(\mathrm{F}(4,56)=2.6343, \mathrm{p}<0.05)$. These results indicate that older drivers, compared to younger drivers, were less accurate at detecting a light change under dense fog conditions when performing two tasks simultaneously.

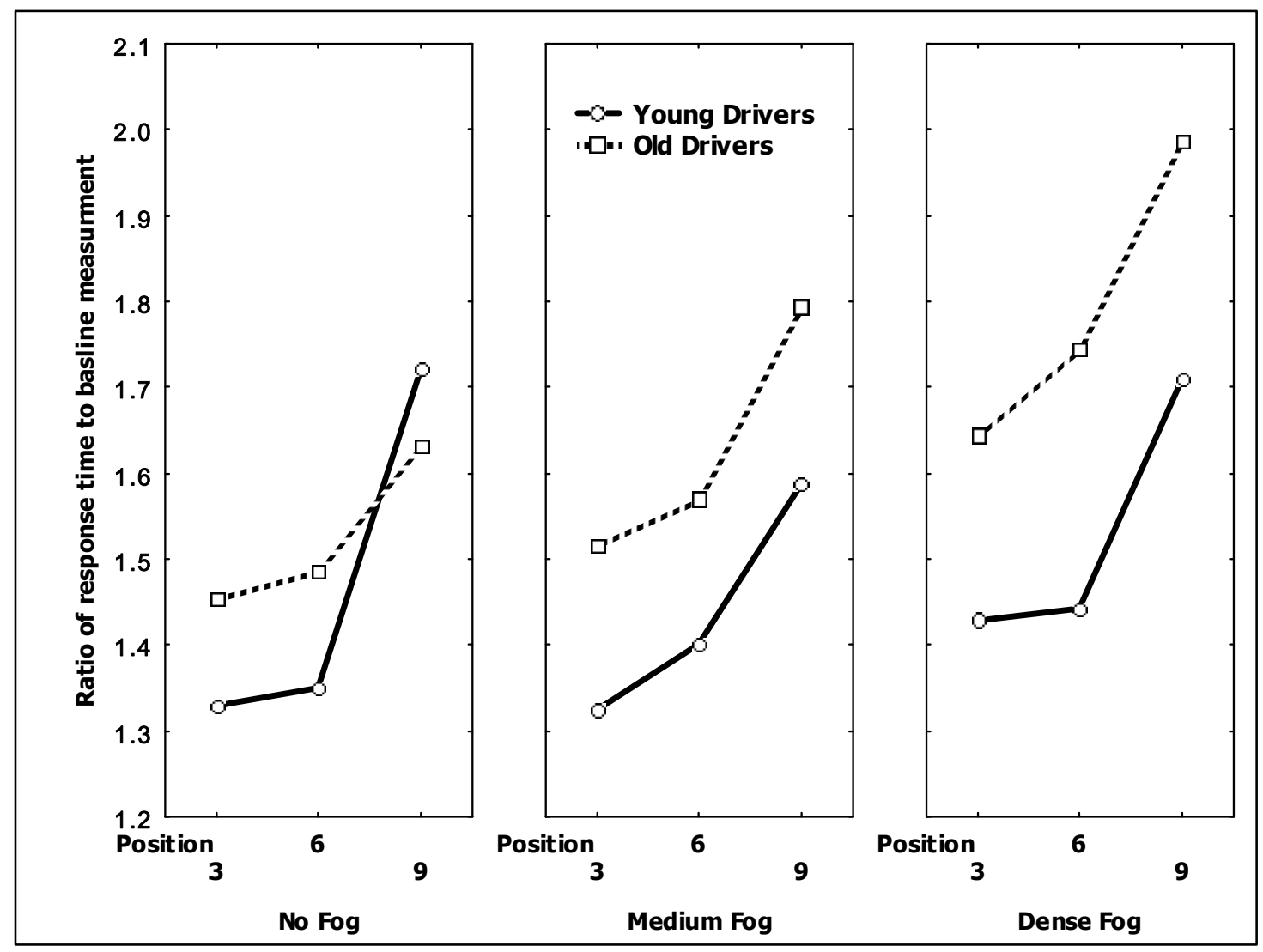

Figure 3. Performance for the light-detection task for younger and older drivers. The dependent variable is the ratio of dual task response time/baseline single task response time. 
With regard to response time (see Figure 3), a significant effect was found for task condition ( $\mathrm{F}(1$, $14)=7.4555, \mathrm{p}<0.05)$, fog density $(\mathrm{F}(2,28)=16.678, \mathrm{p}<0.01)$, and light-change position $(\mathrm{F}(2$, $28)=19.281, \mathrm{p}<0.01)$. In addition, significant interactions were found between fog density, light-change position, and age group $(\mathrm{F}(4,56)=3.6258, \mathrm{p}<0.05)$, and between task condition, fog density, light-change position, and age group $(\mathrm{F}(4,56)=3.0167, \mathrm{p}<0.05)$. These results indicate that older drivers had longer response times to detect a light change under simulated foggy weather, especially when the position of light change was more peripheral.

An ANOVA of car-following performance was performed based on the speed difference between the driver's and lead vehicle (see Figure 4). The main effect of task condition $(F(1,14)=73.903$, $\mathrm{p}<0.01)$ was significant, as well as the main effect of fog density $(\mathrm{F}(2,28)=4.1746, \mathrm{p}<0.05)$. A significant interaction was found between task condition and fog density $(F(2,28)=4.5020$, $\mathrm{p}<0.05)$. Although no significant effect of age was found, we found that older drivers had greater error in following the lead vehicle in the dual-task condition $(F(1,14)=7.2812, p<0.05)$.
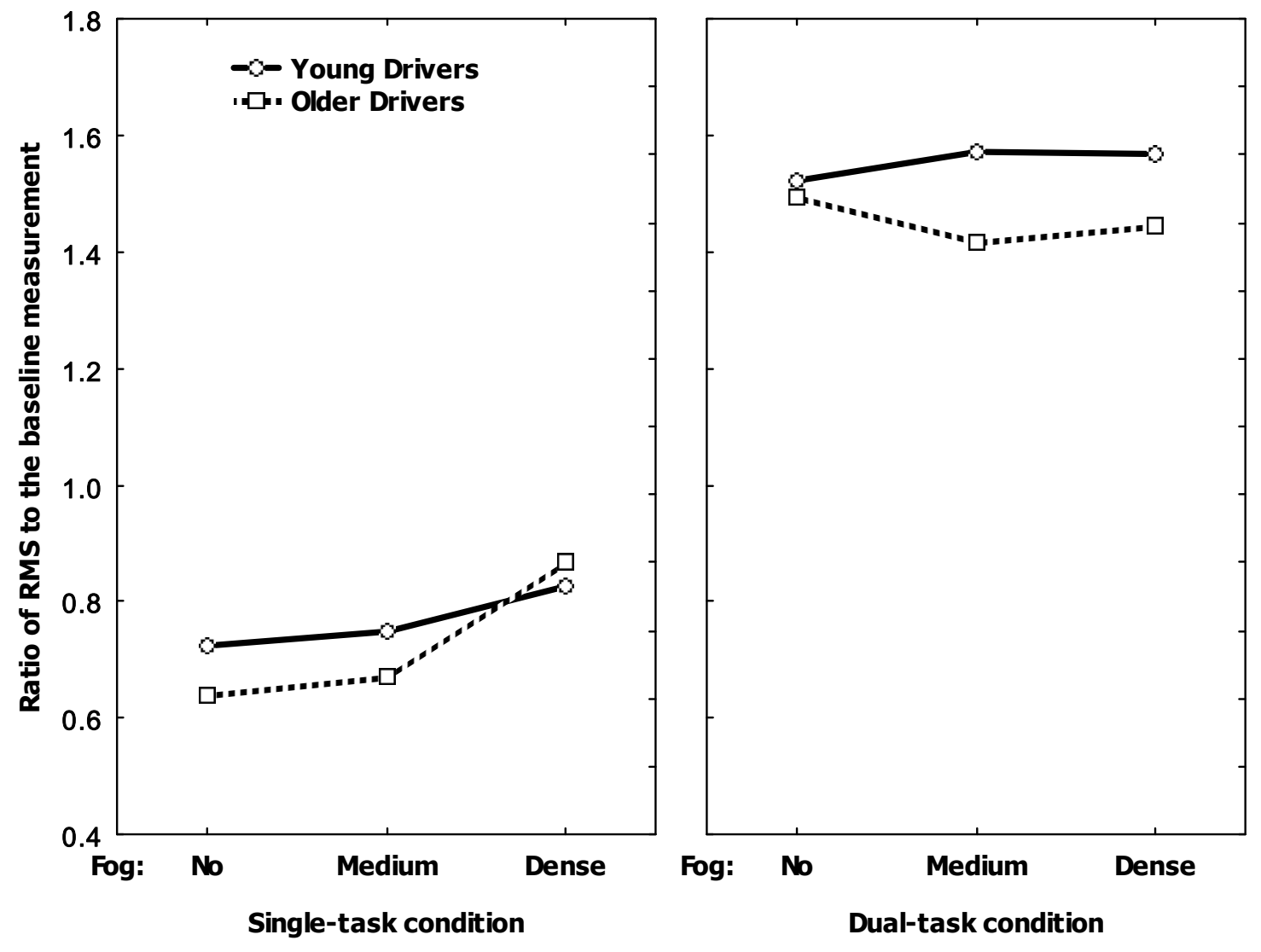

Figure 4. Car-following performance for younger and older drivers. The dependent variable is the ratio of RMS dual-task RMS error/baseline single task RMS error. 


\section{DISCUSSION}

These results indicate less accuracy and longer response times to detect a light change for older drivers, as compared to younger drivers, especially when visibility is reduced. When attending to a secondary task (i.e,. light-change detection) older drivers, as compared to younger drivers, had greater error in car-following performance. These results suggest that under poor weather conditions with reduced visibility, older drivers may have an increased risk of accidents because of a decreased ability to perform multiple tasks.

\section{ACKNOWLEDGMENTS}

This research was supported by NIA grant NIH AG13419-06 and contract 65A0162 from the California Department of Transportation.

\section{REFERENCES}

Andersen, G.J., Sauer, C.W. (2004). Optical information for collision detection during deceleration. In Advances in psychology, Vol 135: Time-to-contact. Amsterdam, Netherlands: Elsevier Science Publishers B.V.

Andersen, G.J, Enriquez, A. (2006). Aging and the Detection of Observer and Moving Object Collisions. Psychology and aging, 21(1), 74-85.

Kramer, A.F., \& Larish, J. (1996). Aging and dual-task performance. In W. Rogers, A.D. Fisk, \& N. Walker (Eds.), Aging and skilled performance. Hillsdale, NJ: Erlbaum, 83-112.

Ni, R., Andersen, G.J., and Rizzo, M. (2005). Age related decrements in steering control: The effects of landmark and optical flow information. Proceedings of the Third International Driving Symposium on Human Factors in Driver Assessment, Training and Vehicle Design, 144-150. 